\title{
EDITORIAL
}

\section{Vorhang auf - Online ist on stage}

\author{
David Kuntz • Johannes Barth
}

Eingang des Beitrages: 6.3.2012 / Online veröffentlicht: 3.5.2012

(C) Springer-Verlag 2012

Früher war der Begriff „Auftritt“ eigentlich eher für das Theater oder die Oper reserviert, aber seit einiger Zeit gilt er auch für das Internet. Natürlich stehen hier keine Tenorsänger im Smoking auf der Bühne und selten klatscht jemand begeistert Applaus. Das holen wir jetzt im übertragenen Sinne nach, denn insgesamt gibt es auf unserer Präsenz im Web eine Menge zu entdecken. Das merkt jeder, der per Zufall nach http://www.fh-dgg.de/ gerät, aber auch diejenigen, die unsere Webseite aktiv nutzen.

Hier werden neben den Angeboten der FH-DGG auch aktuelle Ereignisse angekündigt, welche Arbeitskreise gerade aktiv sind, welche aktiv waren und was es sonst so in der hydrogeologischen Szene Neues gibt. Auch Hinweise für Autoren und Informationen zum Online-Zugriff auf unsere Zeitschrift sind hier zu finden. Des Weiteren stellt die Webseite Formulare und Hilfestellung für Anmeldung und Adressänderung oder auch die Bestellung des Grundwasserkoffers der FH-DGG zur Verfügung. Seit Februar 2012 haben wir unser Angebot um eine Liste der Ehrenmitglieder der FH-DGG, der ehemaligen Vorstandsvorsitzenden sowie Infos zu den durch die FH-DGG verliehenen Preise samt der bisherigen Preisträger erweitert. Ebenfalls findet sich in der Rubrik „Service“ seit kurzem unser Schriftenverzeichnis mit Infos zur Bestellung. Derzeit arbeiten wir an verschiedenen

Dr. D. Kuntz

tewag $\mathrm{GmbH}$,

Am Haag 12, 72181 Starzach-Felldorf, Deutschland

E-Mail: david.kuntz@fh-dgg.de

Prof. Dr. J. Barth ( $\varangle)$

Lehrstuhl für Angewandte Geologie,

Friedrich-Alexander-Universität Erlangen-Nürnberg,

GeoZentrum Nordbayern,

Schlossgarten 5, 91054 Erlangen, Deutschland

E-Mail: barth@geol.uni-erlangen.de
Modifikationen des Auftritts: Unter Anderem werden wir dann auch die Seite IAH-Deutschland unter unserem Dach online beherbergen.

Insgesamt gilt für unsere Webseite: Bravo! Ein beeindruckender Auftritt!

Eine kleine Sache gibt es trotzdem, die man bei unserem Auftritt verbessern könnte: Er sollte keine „SoloPerformance" sein. In der Internet-Szene weiß man, dass ein guter Auftritt auch von der Beteiligung der Gemeinschaft der Nutzer und Fachleute lebt, die Ihr Fachgebiet vertreten. Dann wird er, ähnlich wie die Zeitschrift Grundwasser selbst, ein hochdynamisches Forum für neue Ideen und Trends. Nur schneller und vielleicht auch etwas bunter. Man könnte beispielsweise einen hydrogeologischen Fotowettbewerb starten und die Bilder gleich online veröffentlichen. Oder eine Job- oder Praktikumsbörse online verwalten. Oder ein Diskussionsforum zum fachlichen Austausch zum Thema Grundwasser schaffen. Gute Ideen?! Andere Ideen und Anregungen sind nicht nur willkommen, sondern auch wichtig für die Dynamik unserer Szene. Es gibt viele Möglichkeiten und gilt also ganz ähnlich wie für unseren Nachrichtenteil und unsere Artikel in Grundwasser: Trauen Sie sich etwas anzuregen, $\mathrm{zu}$ berichten, zu vernetzen; wir probieren es gerne aus.

Ihr

David Kuntz und Johannes Barth 